\title{
BMJ Open Social and structural conditions for the avoidance of advance care planning in neuro-oncology: a qualitative study
}

\author{
Henry Llewellyn, ${ }^{1}$ Jane Neerkin, ${ }^{2}$ Lewis Thorne, ${ }^{2}$ Elena Wilson, ${ }^{2}$ Louise Jones, ${ }^{1}$ \\ Elizabeth L Sampson, ${ }^{1}$ Emma Townsley, ${ }^{2}$ Joseph T S Low ${ }^{1}$
}

To cite: Llewellyn H, Neerkin J, Thorne L, et al. Social and structural conditions for the avoidance of advance care planning in neuro-oncology: a qualitative study. BMJ Open 2018;8:e019057. doi:10.1136/ bmjopen-2017-019057

- Prepublication history for this paper is available online. To view these files, please visit the journal online (http://dx.doi. org/10.1136/bmjopen-2017019057).

Received 9 August 2017 Revised 28 0ctober 2017 Accepted 29 November 2017

D) Check for updates

${ }^{1}$ Marie Curie Palliative Care Research Department, Division of Psychiatry, University College London, London, UK

${ }^{2}$ The National Hospital for Neurology and Neurosurgery, University College London Hospitals NHS Foundation Trust, London, UK

Correspondence to

Henry Llewellyn;

h.llewellyn@ucl.ac.uk

\section{ABSTRACT}

Background Primary brain tumours newly affect $>260000$ people each year worldwide. In the UK, every year $>10000$ people are diagnosed with a brain tumour while $>5000$ die annually from the disease. Prognoses are poor, cognitive deterioration common and patients have prolonged palliative needs. Advance care planning (ACP) may enable early discussion of future care decisions. Although a core commitment in the UK healthcare strategy, and the shared responsibility of clinical teams, ACP appears uncommon in practice. Evidence around ACP practice in neuro-oncology is limited.

Objectives We aimed to elicit key social and structural conditions contributing to the avoidance of ACP in neurooncology.

Design A cross-sectional qualitative study design was used.

Setting One tertiary care hospital in the UK.

Participants Fifteen healthcare professionals working in neuro-oncology participated in this study, including neurooncologists, neurosurgeons, clinical nurse specialists, allied healthcare professionals and a neurologist.

Method Semi-structured interviews were conducted with participants to explore their assumptions and experiences of ACP. Data were analysed thematically using the wellestablished framework method.

Results Participants recognised the importance of ACP but few had ever completed formal ACP documentation. We identified eight key factors, which we suggest comprise three main conditions for avoidance: (1) difficulties being a highly emotive, time-intensive practice requiring the right 'window of opportunity' and (2) presence and availability of others; (3) ambiguities in ACP definition, purpose and practice. Combined, these created a 'culture of shared avoidance'. Conclusion In busy clinical environments, 'shared responsibility' is interpreted as 'others' responsibility' laying the basis for a culture of avoidance. To address this, we suggest a 'generalists and specialists' model of ACP, wherein healthcare professionals undertake particular responsibilities. Healthcare professionals are already adopting this model informally, but without formalised structure it is likely to fail given a tendency for people to assume a generalist role.

\section{INTRODUCTION}

Primary brain tumours (PBT) are a spectrum of malignant and non-malignant neoplasms that originate in the brain. They affect

\section{Strength and limitations of this study}

- This study draws together a variety of in-depth accounts from clinical nurse specialists, allied healthcare professionals, neuro-oncologists, neurosurgeons and a neurologist to reveal key social and structural conditions.

- We use the well-established framework method of qualitative analysis, which allows for comparisons to be made across cases and themes.

- By offering a more complex cultural analysis of these conditions, we move the study of advance care planning practice beyond previous descriptions of 'barriers'.

- Our approach shifts attention away from oversimplistic suggestions of recalcitrant healthcare professionals who need training to a fundamental rethinking of advance care planning practice along the lines of a more formalised 'generalist and specialist' model.

- While participants represented a variety of professional roles and range of perspectives, they were all recruited from one specialist tertiary care hospital in the UK and might therefore not be representative of other care settings.

mainly younger people and almost half of those diagnosed are aged under 60 years. ${ }^{1}$ In 2012, 256000 people were newly affected worldwide, ${ }^{2}$ with 10981 new cases registered in the UK in 2014 of which 5092 were malignant. ${ }^{3}$ Prognoses are typically poor, and only $40 \%$ diagnosed with malignant tumours are expected to survive 1 year. ${ }^{4}$ In 2014, 5223 people in the UK died from a PBT. $^{5}$ Brain tumours kill more children and adults under 40 years than any other cancer. ${ }^{6}$

Symptoms are typically multiple, unpredictable and often severe. They include fatigue, motor deficits, decreased concentration, poor short-term memory and speech and language difficulties. $^{7} 8$ Treatments-neurosurgery, radiotherapy, and localised and systemic chemotherapies-contribute further side effects and disruptions, including cerebral 
oedema, 'chemo brain' and the multiple after-effects of irradiation. ${ }^{89}$ These add to the overall burden of a disease that can often affect patients' abilities to make sense of themselves and others. The disease may also interfere with patients' decision-making processes and higher level executive functioning. ${ }^{9-12}$ For some, mental capacity and cognitive ability may fluctuate while others experience a steady decline.

Given this complex clinical picture and existentially threatening context, brain tumour care and treatment, especially with regard to the future, is extremely diffcult to navigate. ${ }^{13}$ Accordingly, patients, their families and healthcare professionals are advised to begin early and ongoing discussion about their care needs, especially towards the end of life (EOL). ${ }^{14-16}$ Policy documents and neuro-oncology communities recommend the early introduction of advance care planning (ACP) to establish these discussions in routine care. ${ }^{17} 18$ The main aim of ACP is to clarify a person's wishes in the anticipation of a physical deterioration that might cause loss of capacity to make decisions and/or compromise their abilities to communicate wishes to others. It is defined as a process of discussion between an individual and their care providers and may include both family and friends. ACP can also incorporate more formalised advance statements of preferences and wishes as well as legal processes such as lasting power of attorney and advance decisions to refuse treatment. ${ }^{19}{ }^{20}$ It is recommended that plans should be documented, regularly reviewed and communicated to key persons involved in patients' care. Importantly, ACP is considered to be the 'shared responsibility' of the multidisciplinary team (MDT), where any healthcare professional, 'regardless of discipline', can engage in its practice.

Although widely advocated in policy documents, ACP is not without critique. Some research has questioned whether ACP improves the delivery of care at the EOL, ${ }^{21-24}$ while others note its absence in routine clinical practice. ${ }^{25}$ In accounting for this absence, these studies list 'barriers' such as the timing of the discussion, lack of knowledge, skills and training ${ }^{24} 2627$ and a perceived unwillingness of patients to engage in discussions about EOL. ${ }^{28}{ }^{29}$ While useful in identifying some of the challenges in ACP, the focus on studying 'barriers and facilitators' has assumed the presentation of particular isolated factors with the intrinsic power to impede or promote practice. This fails to connect barriers to broader structural conditions and assumes that by simply removing the barriers, practice will spontaneously change. This literature has also tended to characterise healthcare professionals, particularly nurses, as recalcitrant and in need of training, without considering a more sophisticated view of the complexities of care. ${ }^{2629} 30$

Currently, there is limited understanding of the contexts in which 'barriers' develop or how they relate to the social and cultural dynamics of the healthcare environment. Moreover, most ACP literature focuses on general chronic and terminal disease or specific disease groups other than brain tumours; there is very little research accounting for the specific condition of brain tumours. ${ }^{17}$ Given the natural history of brain tumours, the fact that they often affect younger people and thereby challenge assumptions about normal life course, and challenges to cognitive status as the disease unfolds, more focused research is needed.

In this article, we use in-depth qualitative methods to elicit key social and structural conditions that contribute to an observed avoidance of ACP practice by professionals working in neuro-oncology. We define these conditions as the underlying social, organisation and technical factors 'that shape the nature of situations, circumstances, or problems to which individuals respond by means of action/ interaction/emotion,. ${ }^{31}$ Rather than simply being an absence of practice, we take the avoidance of ACP to be a social event, which is dependent on a complex set of social practices. In so doing, we hope to provide a more nuanced understanding of ACP in practice and deflect some of the negative attention away from healthcare professionals who have become the fall bearers of the avoidance problem.

\section{MATERIALS AND METHODS \\ Design}

Cross-sectional qualitative study using in-depth semi-structured interviews.

\section{Sample}

To gain a range of experiences, we purposefully sampled healthcare professionals working with people with PBT from one NHS foundation trust in London, UK. We aimed to include professionals from all disciplines with a variety of experience working in neuro-oncology. Fifteen individuals were identified and invited to participate via email by clinical members of the research team (JN, LT). All agreed to take part.

\section{Data collection}

HL, a trained ethnographer with a social science background, conducted one-to-one in-depth semi-structured interviews with each participant using a topic guide, based on themes from the ACP literature and expert opinion, to explore participants' assumptions and experiences of ACP (see figure 1). Interviews lasted between 50 and $165 \mathrm{~min}$, and were audiotaped and transcribed. Transcripts were imported into NVivo (V.10), a qualitative analysis software package that supports data coding, management and the production of data matrices. ${ }^{32}$ Interviews took place in a quiet room in the hospital where participants worked.

\section{Data analysis}

All transcripts were analysed using framework analysis. ${ }^{31}$ First, transcripts were open-coded independently by two researchers $(\mathrm{HL} / \mathrm{JL})$. Second, codes were judged for connections and grouped together to develop a thematic framework. Third, data matrices were built on this framework with themes running across columns and participants running down rows. Fourth, data corresponding to these codes were inputted into the matrices. Matrices thus provide a data management tool through which data 
- Their roles in caring for patients with primary brain tumours, particularly those with high glioma.

- How they would approach conversations around future care.

- Their perspectives on the emotional and existential aspects of engaging with patients about their futures.

- Their understandings of patients'and families' current and future needs.

- Their understandings of the concept of advance care plans and their familiarity of making advance care plans.

- Their understandings of documenting advance care plans.

Figure 1 Topics explored in interviews with healthcare professionals.

were analysed both across participant and across theme. ${ }^{33}$ Throughout analysis we attended particularly to assumptions about ACP, factors making ACP difficult as well as the contexts of routine work and perceived professionals' identities. Drawing these together, we suggest the social and structural conditions that shape the nature of the situations, circumstances or problems ${ }^{31}$ that characterise an avoidance of ACP. The idea of avoidance emerged within participants' accounts but it was also something we were especially sensitive to given our understandings of the literature. To illustrate the inter-relationships between these conditions in the production of avoidance, we modelled them on the commonly known fire triangle (see figure 2). The fire triangle is a model representing the conditions needed for fire-fuel, oxygen and heatand hence is a useful reference point for how we might think about the social and structural conditions for avoidance. Transcriptions were not returned to participants. To ensure interpretative validity and meaningfulness, themes identified were discussed with clinical members of the research team (JN, LT, EW).

\section{Ethics}

REC approval was not required as health service ethics is not required for interview studies with health service healthcare professionals. All participants gave written informed consent.

\section{RESULTS}

Our final sample included 15 healthcare professionals: 4 neurosurgeons (mean age 53, mean years experience 21.5 (range 10-32), 50\% female), 3 neuro-oncologists and 1 neurologist (henceforth 'physicians' for anonymity) (mean age 50, mean years experience 16.5 (range 10-26), 50\% female), 4 clinical nurse specialists (mean age 38 , mean years experience 4.6 (range 1-9), $100 \%$ female), 1 occupational therapist, 1 physiotherapist and 1 speech and language therapist (mean age 32, mean years experience 3.7 (range 1-7), 100\% female) diverse with regard to years of experience, gender and age.

Most participants recognised the importance of having conversations about future preferences for care with patients. However, they perceived the delivery of ACP as challenging with very few having ever completed an ACP in documented, structured conversations. In our analysis, we identified a number of key and overlapping themes, which we organise into conditions: (1) difficulties, (2) presence and availability of others and (3) ambiguities. We suggest that combined, these conditions created what we call a 'culture of shared avoidance'. In our adapted 'fire triangle' (figure 2), each triangle represents a condition necessary for the culture of shared avoidance. We describe these conditions in turn before describing how they combine

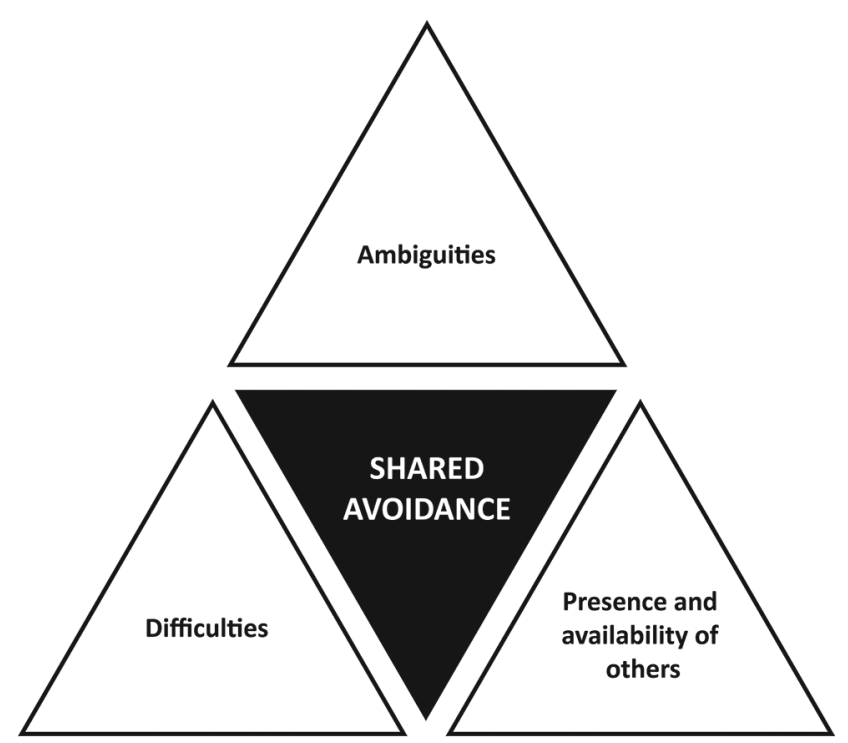

Figure 2 The culture of shared avoidance. This model uses an adapted fire triangle to represent the interaction of the three conditions that produce avoidance. We call this the culture of shared avoidance. 
to create a culture of 'shared avoidance'. Short excerpts and long-form supportive quotes are included in box 1-3, allowing first person narratives to be presented.

\section{Condition 1: difficulties}

This outlines the specific challenges that participants identified as a condition for avoidance by focusing on descriptions of their difficulties with ACP. It also presents features that participants thought important to good ACP practice. Supportive quotes are displayed in box 1 .

\section{Emotive conversations}

Participants across specialty felt that ACP involved discussions around emotive and existential issues concerning illness, dying and mental capacity, which needed to be raised sensitively. They described difficulties in conveying information about disease prognosis to patients, especially if this was negative, and feared being 'out of their depth' and upsetting patients. This difficulty was further compounded by the relatively young age of patients and the sense that this group wanted to engage in conversations about treatment.

\section{Lacking time and patient contact}

It was commonly thought that ACP discussions required time and patient contact. Yet time was a scarce resource in the context of a busy clinical environment. Some described this lack in contexts of having to account for their work and the difficulties in having ACP recognised as a legitimate activity. As a result, ACP slipped down lists of priorities, relegated to ad hoc moments when participants found themselves with a spare moment. Time was also implicated in discussions about establishing rapport with patients and families. In this way, it was spoken about in the context of patient contact and being able to develop the necessary level of intimacy for good ACP practice. Many thought that they had not had enough time with individuals to be able to establish such rapport.

\section{Windows of opportunity}

The timing of these sensitive and complex conversations was also thought to be key to good ACP practice. So rather than simply having enough time, this was about finding the right time. Participants described 'the right moment' or 'window of opportunity' for ACP. These were often characterised as times when patients had begun to understand the implications of their disease and when they would be more receptive to considering EOL. Importantly, these moments also required patients to be able to engage in discussions. Such moments were thought to be rare, and especially so early on in patients' trajectories given that care is often framed around intervention and the intent to treat. While most agreed that these conversations needed to happen early on, the 'windows of opportunity' were hard to find. Instead, participants described waiting for the medical or social situation that required them to act. Given the rapid and unpredictable nature of brain tumours and issues with capacity, participants described how windows often closed soon after opening. Some did suggest ways in which the window might be prised open but did not necessarily practice these suggestions.

\section{Condition 2: presence and availability of others}

This highlights the presence and availability of others as a condition for avoidance. It rests on ideas about appropriateness and assumptions about professional remit - whose job is it and who projects the appropriate professional identity. It also rests on notions that ACP be done by someone skilled, confident and compassionate. Supportive quotes are mentioned in box 2.

\section{Professional remit}

Professional remit was a common way for participants to mark out and designate responsibility. Neurosurgeons and physicians, for example, tended to frame their work by its focus on treatment. They saw patients at particular times during the illness trajectory-typically moments of intervention-and they did not see ACP in their remit. Instead, they tended to designate ACP as nurses' work on the basis that nurses had more time and better rapports with patients and that nursing incorporates the 'softer aspects of care' like discussions about the future. Nurses similarly used remit to shape responsibility and position themselves vis-à-vis ACP, but in a different way. They considered ACP as being foremost about EOL and hence part of a palliative care remit. While more nurses prepared to engage in conversations about the future, they often positioned themselves as 'signposters', assessing needs and referring on.

\section{Professional identities and perceived expectations}

Relatedly, participants made assumptions about how patients perceived their roles by assuming that patients shared in their conceptions of remit. Accordingly, some suggested that patients might be confused if they or others brought up care at the EOL. This was related to shared investments in hope and concerns that introducing conversations about EOL would disrupt more optimistic narratives of care.

\section{Personality and rapport}

It was not simply on the basis of formal remit and perceived role that participants designated what we might call the 'appropriate other'. They also used less tangible attributes to do with personality or skill. Some named particular people who had abilities to get patients to open up and talk, who did not feel awkward during emotive conversations or being direct in raising difficult issues. These abilities were not bound to role or remit but were intrinsic to the person. Participants would also identify and refer on to those who had had the opportunities to develop rapport with patients and families and who therefore were better placed to initiate and steer ACP.

\section{Condition 3: ambiguities}

This outlines the condition of ambiguity given in participants' conceptions of ACP-the notion of shared responsibility and what it is that delineates ACP from other care 
Box 1 Supportive quotes for condition one: difficulties (emotive conversations, lacking time and patient contact, windows of opportunity)

\section{Condition 1: difficulties}

Emotive conversations

"I know this is so ridiculous, but I just don't like it when they get upset too much, and when it's something that I have absolutely no idea how to help them with. I know you don't need to say things all of the time, and sometimes they just want to cry, which is fine. But I feel really helpless. And they are going to die, and it's a shit condition and prognosis, and they're going to die in a very undignified way —not always, but most of the time. It's very difficult. And you know when you just think, 'Yeah, it is. That's unfortunate'. There's nothing you can say. It is what it is. Sometimes I just think, 'What else can I say to that?' This is it. Everyone is going to be torn apart". (Clinical nurse specialist)

"I think it's just good that I know my limitations. I just don't think I want to do something that I can't do well when it's this emotional and raw. You need someone with confidence". (Clinical nurse specialist)

"It's both hugely emotive and time consuming to engage in these conversations. It's quite intensive work, which needs to be done by people confident in having those conversations. And again, that's not everybody". (Clinical nurse specialist)

"I think you could probably level the criticism at neurosurgeons that we are a bit more emotionally removed from our patients than perhaps in some other specialities. Because we have to deal with, as I say, direct consequences of what we do and some of those are devastating and that's quite a difficult thing I think to deal with emotionally, particularly if you get hugely, hugely overemotionally involved with every single patient that you see. That's hugely emotionally costly. And I also think not only is it emotionally costly, I think it also actually stops you seeing the wood for the trees and therefore actually really doing the best thing for the patient because you start thinking about them as, you know, 'Oh, they really remind me of my mum'. As soon as you've done that it's your emotions talking rather than actually the brain and, you know, the intellect speaking. Does that make sense?" (Neurosurgeon)

Lacking time and patient contact

"It's hugely emotive and hugely time consuming to engage in these conversations. So bearing in mind we're in an environment at the moment where everybody wants to know that what you are doing is either generating income or cost effective, justifying that amount of time on having a conversation to enable someone to come to a good decision, I know it's good quality care, but that's in amongst 101 other things that also have to be done which are more clinically measurable, have a better outcome in terms of ticking a different type of box". (Clinical nurse specialist)

"Time-it's always about time for me. Because those (patients for whom ACP relevant) are the people that l'm seeing in outpatients and occasionally they will come in for an operation because that's how you're going to palliate them. But at the time of the operation what you're worried about is the immediate operation and implications of the operation and getting the patient through the operation, making sure they're recovering and then you're trying to get them home. You're not focusing on the longer-term issues. You're dealing with an outpatient context and a surgical context. I run an hour late in my clinic if I'm lucky, so there are times when I feel frustrated by that. You know, I try quite hard to give the time to the patients that I think they need but sometimes you're aware of thinking, 'Actually, although I need to start talking about this I just don't have the time because l'm an hour late and if I start this discussion it's not an easy discussion to do. It could be half an hour'. And quite often in that situation what I will do is to refer them to the clinical nurse specialists, if I can. So that extra resource is also really useful. So, yes, that's often my compromise, if you like. And it's not always because it's the coward's way out, you know, from having a difficult discussion. Some of it is about, as I say, actually the sort of practical terms and practical issues. Wanting to make sure that somebody has got enough time to discuss those things and to think about those things without feeling rushed-without me sort of hopping from foot to foot because, actually, now l'm an hour and a half late in my clinic. So time is the really big issue for me". (Nurosurgeon)

"The clinical nurse specialists can often help be that person who communicates between family and the team. They're really well placed as they're likely to follow the patients up afterwards as well. It's important that the family have that key person that follows them onwards". (Clinical nurse specialist)

\section{Windows of opportunity}

"The difficulty for the brain tumour patients is that you may only have a window of time where they have the cognitive ability to actually address some of these issues". (Clinical nurse specialist)

"It's not that there aren't opportunities, it's just the way our clinics run. It's quite a difficult thing to do (ACP) when you're concentrating on getting on with treatment and potential problems related to treatment rather than totally diverting the attention from that on to, 'Have you thought about the future, and what you're thinking about doing in the future?' It just seems not appropriate in these sorts of clinic appointments". (Physician)

"I mean, there are other things that are problematic with this patient group in that sometimes the person to whom it's happening, who's got some cognitive impairment, has absolutely no insight into it. So although they have capacity to initiate a Lasting Power of Attorney, they could rationally think about it and make a decision to do it, they don't detect that they have a problem. But you couldn't then force one on them because they do have capacity. So there's a very grey area, very, very grey area with regard to their insight into the problem. If that's part of the cognitive process that they can't see that they are behaving a bit strangely, or that they are not willing to accept that there will come a time where they can't act for themselves, be that because they are no longer able to think straight, or verbally communicate, or that they are moribund in a bed unable to wake up to do stuff". (Clinical nurse specialist)

“Well, I think it's partly just we don't because it doesn't arise, and also we don't really want to bring that into the picture when actually the patient might not want to discuss it. But perhaps if we had more of a formula for when we first see someone, these are the things we should make sure we discuss, then that might be easier. Because I often feel, you know, certainly when I used to do clinics with (the palliative care consultant) and she used to come along, we would often go, 'Oh, no, we can't discuss that with that person, can we? It's just not appropriate, it's going to be too upsetting for them', which obviously you don't want to upset people. But if it was more of our routine and we explained to the patients, 'Look, we hope this isn't going to be happening in the imminent future, but we think it will be sensible to discuss this with you whilst you're well. Would you mind?' And if we said, 'We do this with absolutely all of our patients', I think that would be less of a problem maybe. I don't know". (Physician) 
Box 2 Supportive quotes for condition two: presence and availability of others (professional remit, professional identities and perceived expectations, personality and rapport)

\section{Condition 2: presence and availability of others}

Professional remit

"So normally we have a conversation about their treatment. If I'm pushed, then I will tell them that the average life expectancy with this grade of tumour is 1 year. Most patients get very upset when you tell them that, so I try to avoid that to be honest, at that stage. I leave the patient with the clinical nurse specialist, because they're much more experienced in managing the practicalities of what's going to happen to the patient next".

(Neurosurgeon)

"A surgeon is like a technical person, who will do the (surgery) and give a plan, and that's it. If you're doing an operation then I don't think you should be the primary person, because there are other skills which are needed, with people trained in that area, better than a surgeon. They have more patience. Basically a palliative care nurse, a supportive care nurse or a consultant, they have for more patience than a surgeon would have, without question". (Neurosurgeon)

"And sometimes that's better done by not me, but by the CNS or someone very much more patient-friendly than a doctor. First off, they have more in-depth day-to-day knowledge of how a patient will be and how they will feel, because they acquire that skills through their experience in supporting and caring for lots of different patients". (Neurosurgeon)

Professional identities and perceived expectations

"The patients will expect us to be talking about (medications), whereas talking about ACP they probably think, 'what on earth was he doing that for today?' It might be worrying for them. They might think, 'perhaps he knows something I don't'." (Physician)

"Patients come to see me after they've just had a scan, so the first thing they want to know is, 'What's happened to my tumour, what's happening on the scan?' So the appointment is focused on the immediate needs of the tumour, of treatment". (Physician)

"There will always be an obvious person who as part of that team has an obvious opportunity if that window opens up. With the high-grades there's a very clear transition from speciality to speciality. So the high-grades are in their surgical episode to start with, then they move on to their oncology episode and that window isn't in that surgical episode, it's in the oncology. Whereas there are other patients they're seeing the oncologist and the surgeon alongside each other and I think, you know, in terms of bringing it up as a discussion it should be a, it's a combined responsibility". (Neurosurgeon)

Participant: "We may not have a specific conversation about advanced care planning but we often very much right from the beginning have already liaised and very much communicated with the clinical nurse specialist on, you know, 'This is what they've said to us, this is what they want, this is where they're at'. And that's a continual process. Within that, they may say, 'Well, they're looking at advanced care planning', and you can say, 'Well, actually, they've said this to me, they've said that to me, they've said this'. So it's sort of giving them that information".

Interviewer: "So, I mean, it sounds like from that perspective you're very much involved in those sort of things but you might not necessarily be sort of talking about it in those terms".

Participant: "Actually we are but we haven't sort of labelled it as such".

Interviewer: "Yeah. And is there a sort of a push for you to begin to label things like that or do you think-".

Participant: "—Not that I'm aware of. I know that we're very much involved in all aspects of the process but not that l'm aware that specifically, and this is where roles blur, it's knowing who is sort of the driver of the advanced care planning, as it were".

Interviewer: "And who is that?"

Participant: "In my opinion, and this is where l've only seen it in action on this ward, the palliative care team tend to lead those discussions and it's whether they are highlighting it to us and we have missed something or, I don't know, I just find that the palliative care team tend to lead on that". (Allied healthcare professional)

Personality and rapport

"I don't have too many conversations about end of life, but it does happen. Those are very difficult, but I feel confident I can do those conversations now, because l've had so much experience. I see where treatment is beneficial and where it's not. I never say there's no hope, but I try to explain that the focus of care is now shifting onto, 'Keeping you as comfortable for as long as possible and managing any distressing symptoms'." (Physician) "It's difficult to say, because all neurosurgeons are going to have different personalities. But I think most neurosurgeons would not like to be involved in the advance care, supportive care, as much". (Neurosurgeon)

Participant: "I think that's partly, you know, neurosurgeons are perhaps a specific type of personality and, you know, everybody has their vocation I guess and their speciality that they're best at and that's why you end up in it. But there's also the other side to it that means that you may not be as good at doing some of the other things that you have to do as part of your role".

Interviewer: "Because you hear of these sort of stereotypes, don't you, of the neurosurgeon".

Participant: "Yes".

Interviewer: "I mean, could you tell me a bit about that-when you say the personality of a neurosurgeon?"

Participant: "Well, yes. I mean, one does generalise and clearly in many ways I can only really talk about me and I'm not sure if people think that I'm a normal neurosurgeon. I'm not sure if that's a good thing or a bad thing but anyway. I think we do deal with catastrophes and we do deal with catastrophes that we have created. And so from a very personal level, I'm aware that, certainly when l'm operating, I'm not thinking about that individual at an emotional level because I can't because I think that then stops me from doing what is the right thing for that patient intraoperatively. That's not to say that l'm not emotionally engaged with them when they become a patient again, when l've taken the drapes off and put them back together again". (Neurosurgeon)

"As therapists, I think, we are generally better at communication, I don't know whether it's just the type of person that would choose to be a therapist. But I know that's not the focus with the other than obviously palliative care doctors. The palliative care consultant is just phenomenal, the best communicator ever but she's a real anomaly in the medical profession". (Allied healthcare professional) 


\section{Box 2 Continued}

“Well, we've (healthcare professionals) all had to do the communication skills course. I think it is just part of you and you can either do it or you can't. Maybe that's just because they (the doctors) don't want to open a can of worms I guess, so they leave that to others". (Clinical nurse specialist) "Yeah, so l'm not very good with the whole psychological part, really. So if things do come up, I get (someone else) in (...) Things like death and the nitty-gritty bits about what actually upsets them. I'm not very good at getting to the actual part. I'm not very good at it. So that's when I call (someone else) in (...) I try to follow it through, but I can't. I kind of just flap around instead. (A named consultant) is good because the way she asks questions are quite direct, and how she approaches subject. Obviously she's very experienced, that's what she is a consultant for. But I just don't do that". (Clinical nurse specialist)

activities. In many ways, it is the most fundamental condition-a point to which we return in the next section. Supportive quotes are mentioned in box 3 .

\section{Constitutive practices of advance care planning}

The specific practices that constituted ACP were unclear for many participants. While they understood its key tenets-early discussion, future care, end-of-life-they did not necessarily understand how it was different from much of their other work. This was particularly salient for nurses and allied healthcare professionals who already engaged in practices that touched on end-of-life and planning for the future. Discharge planning and occupational health assessments were given as comparative examples, both of which involve formal needs assessments, the anticipation of decline and documented outcomes. Whether these constituted ACP was debatable. Participants recognised how such practices might contribute to a conception of ACP that is distributed among the clinical team, and as such acknowledged their indirect role in planting seeds for later conversations about future preference for care. Yet, they were equally clear that the main focus of these practices was the immediate need to get patients home safely following inpatient admission or to refer to appropriate services.

\section{'Shared responsibility'}

Many participants recognised that ACP was a practice of 'shared responsibility'. Yet what this meant was unclear and they questioned what exactly it was that they themselves were responsible for and how their role fitted into ACP. Again, this bears on conceptions of ACP as a distributed process and for some this seemed to run counter to the boundaried work characteristic of medical specialisation, where core responsibilities are clearly defined and delineated. Unlike these core responsibilities, participants did not think themselves accountable for ACP - they assumed that someone else would be doing it and it was not something for which they needed to take responsibility. Multidisciplinary team meetings were seen by some as a forum where shared responsibility for ACP might be negotiated. Yet they suggested that the intent to treat dominated these forums and closed such possibilities.

\section{A culture of shared avoidance}

This section draws together how the three conditions identified above combine to produce avoidance. We use the fire triangle by way of explanation. The fire triangle is a model representing the conditions needed for fire-fuel, oxygen and heat. A fire occurs given the right combination of these conditions and this helps to understand fire as an event. Following this model, we might substitute the event of fire for the event of avoidance, and the conditions of fuel, heat and oxygen for the conditions of difficulties, presence and availability of others and ambiguities (see figure 2). When this event is sustained over time, this produces a culture of shared avoidance. It becomes a self-perpetuating state that is fuelled and furthered by the continual interaction of the three conditions. The positive characterisation of event helps to capture the productive aspects of avoidance. That is, avoidance is not merely an absence of action but the result of cumulative actions over time.

This model draws our attention away from thinking about these conditions as isolated 'barriers' - as things that have some intrinsic properties which prevent ACP from happening. Instead, it highlights how conditions are activated in their relationships to other conditions. When we think about the difficulties that participants perceived in doing ACP, for example, we do not simply see difficulties as barriers per se, but difficulties as a condition for avoidance given in the context of other social and structural phenomena. In this case, we see the presence and availability of others and ambiguities in the definition of ACP as conditions which activate difficulties. In essence, participants were able to avoid ACP because they thought someone else would do it and they were unsure about their specific role in the process. Similarly, the presence and availability of others is itself not a barrier, but a condition for avoidance in its relationships to perceived difficulties and ambiguities in the definition of ACP. Here, we suggest that participants did not simply avoid ACP because other people were available to do it, but because they also found it challenging, were busy and did not see themselves as accountable for doing it.

This approach therefore highlights how difficulties, presence and availability of others, and ambiguities are not barriers to ACP per se but conditions for avoidance given in their relations to other conditions-they are therefore embedded in a broader culture of avoidance.

\section{DISCUSSION}

As with previous studies, we suggest that ACP is often perceived as peripheral to the work of healthcare professionals working in tertiary care and for whom the dominant 
Box 3 Supportive quotes for condition three: ambiguities (constitutive practices of ACP, 'shared responsibility')

\section{Condition 3: ambiguities}

Constitutive practices of ACP

"So you start having those discussions (ACP). I don't have the skills to properly delve into that from an emotional point of view. Probably I would look at it more from a practical point of view, about how we can facilitate it (home discharge) and increase the safety, rather than sort of the other side of things really". (Allied Healthcare professional 10)

"I guess we are (involved in ACP), so that's a lot of what the community planning is. I think, especially for the higher grades (of tumours), with any type of community or palliative care involvement, you are putting steps in place, that people will get involved in anticipation of what's to come. So from a therapy perspective, you're trying to set them up in anticipation. But I don't really understand exactly what advanced care planning is. It's quite a foreign thing for therapists to be involved in". (Allied Healthcare professional 9)

"Well because we're always thinking discharge, and how we can get them home as best we can, and setting them up, to a certain extent, you are probably doing some of the care planning there and then, and by involving palliative care earlier, hopefully we're instigating steps towards all that planning". (Allied Healthcare professional)

'Shared responsibility'

"If you are the surgeon who has operated, then you should be involved in that advanced care planning, although your role may not be as much as the role of a palliative, supportive care consultant. So once (the surgeons) have done their bit, the supportive care consultants should be the lead, but you should still be involved". (Neurosurgeon)

"I guess it's from the start (doing ACP), and everyone should be doing it to whatever capacity, planting these seeds in patients' heads. Because you don't know who they've met before (referring to healthcare professional) and what conversations they've had". (Clinical nurse specialist) "We may not have a specific conversation about advanced care planning but from the beginning we have already liaised and communicated with the clinical nurse specialist on, 'This is what they've said to us, this is what they want, this is where they're at'. And that's a continual process. Within that, they may say, 'Well, they're looking at advanced care planning', and you can say, 'Well they've said this to me'. So it's giving them that information". (Allied Healthcare professional)

"So I do probably see more of a role now, in advanced care planning, but truthfully I don't understand what it actually is and what it entails. Hopefully not us, because if that's the case l'm not doing anything! I always think it's under palliative care. Whether that's right or wrong I don't know". (Allied Healthcare professional)

"Palliative care is a sort of term that sort of encompasses everything, both rightly and wrongly. And I suppose when people are referred to palliative care, the surgical teams or the oncology teams might make assumptions about what they are going to do. And in essence that is what they do, which is why it becomes so difficult to refer patients to palliative care because they also have the fear that this just means talking about death, which, of course, isn't all it is, but a lot of the time it is talking about death. So then there's a reticence sometimes for oncologists to refer to palliative care because they don't feel their patients are at that stage and neither do the patients, and yet the oncologists aren't having the conversations around advance care planning and neither are the neurosurgeons. (...) There's this limbo. I think it is a really uncomfortable zone. I mean everyone is hoping someone else is going to do something about it. (...) This notion that patients are on care pathways, and everybody knows where they're at, and all the right people are involved - it's good that that's what everyone is striving towards, but the reality is that it isn't happening very often. It certainly isn't happening in the practice that l'm in. So coming to something like thinking about us as a team working towards implementing advance care planning, I just can't see how it could happen without maybe some of the other elements of working as a team being improved. And communication, communication is key, so all the stuff that has come up about, 'How do you know it's been done, how does somebody else know what's been said?' Without knowing those things, it's really hard to carry something forward. And it can't be the responsibility of just one person in the team, because they travel between clinicians. Even if they come to clinic under the same consultant, they might see a registrar one week, and then six month later the registrar has changed, there's a new registrar. So they come and they don't always see the same person. So unless it's very clear that that conversation has been had, there's no way of knowing where that patient is. So communication is probably key, and maybe just finding a way to get people to communicate about whether or not a discussion has been had and what the content of it was, and there being in place for people to be able to go and access that would be a beginning to actually clinical teams being aware of the information pathway that the patient was on. But we just don't. I know from my patients, the conversations that l've had with them... but... and if l'd done something like an advanced directive- which I never have, because no one has asked for one-I would tell my team members. But it really just doesn't happen. So I don't know. That's it, I can't say anything else". (Clinical nurse specialist)

driver of care is the intent to treat. Similarly, we have identified several difficulties with ACP which resonate with the 'barriers' identified by previous studies. ${ }^{24}$ 26-29 These include, the perceived lack of confidence and skill healthcare professionals have in engaging in emotive discussions. We also highlight the busy healthcare environment, the difficulties healthcare professionals have in identifying the right moment to do ACP and the overall lack of time allocated to aspects of care lacking an observable legitimacy. The particular context of brain tumours is pertinent here given the suddenness of change that can occur in patients' capacities to engage in care decisions and the tragic mysteries surrounding mental capacity. What is more, is the pervasiveness of the treatment imperative and the fears associated with disrupting narratives of hope endemic to cancer. ${ }^{34}$ While this is particular to cancer and brain tumour communities, these themes also emerge out of wider cultural ambiguities around what it means to die well and the roles that should be taken by medical intervention. ${ }^{35-38}$ In these windows of opportunity, we see how healthcare professionals' attitudes are in a fluid and dynamic interaction with the views of patients and their families including their willingness to talk about future care and the prospect of dying. 
This study shifts the emphasis of previous studies which have tended to assume that difficulties, or 'barriers' in their vernacular, are located within the confines of individuals, either through healthcare professionals lacking confidence or skill to do ACP or patients' 'unwillingness' to engage in discussions about death. Rather, we take an approach that sees difficulties to be embedded in larger structures. As such, we consider them to be conditions for avoidance constructed and activated in their relationships to other conditions, namely the presence and availability of others, ambiguities in what constitutes ACP and the notion of 'shared responsibility'. This approach therefore gives a more nuanced account of a broader 'culture of avoidance'.

At the same time, we acknowledge the methodological limitations of our work as our results are based on healthcare professionals working at one specialist centre in the UK. These perspectives may not reflect the perspectives of healthcare professionals working in other sites with a less specialist interest in neuro-oncology, or in other countries, where both health policy and healthcare delivery may differ substantially from the care delivered in the UK. Moreover, as with much qualitative work which focuses more on the 'particular' over the 'general', our sample size is small and does not include representation from other professional groups who are also implicated in ACP, such as social workers. While we have presented some of the ways in which participants reflected on things like professional identity and personality, a systematic analysis of the determining of effects these factors and others, such as age, gender and experience, was not the intended purpose of our study.

\section{Implications}

There are several important implications from our findings. First, any intervention that attempts to increase the potential for ACP in primary brain tumours must be multifaceted and attend to the multiple conditions of avoidance. Training, such as advanced communication programmes, ${ }^{39}$ may be important in supporting healthcare professionals to overcome difficulties, develop confidence and skill and helping them to identify and affect windows of opportunity. However, training is simply not enough on its own, and without more complex interventions addressing the social and structural conditions we highlight, avoidance is likely to persist. In particular, it is important to ensure that organisational support is in place in clinical practice.

Second, a formalised 'generalist and specialist' model of ACP might be an appropriate way to disrupt the ambiguity of 'shared responsibility'. Healthcare professionals are in some ways already doing this by referring on to those with the right personalities of skills, but without structure it will fail because everyone assumes the role of generalist. Here, certain healthcare professionals would be ascribed particular roles and responsibilities that would be delineated in codes of conduct and set within a formalised pathway to ensure patients are routinely offered ACP. This pathway would unfold as a continuous discussion beginning at the point of diagnosis and responding to the clinical situation.
There would also need to be some accountability structures and through this recognition that ACP is a legitimate practice requiring time. Finally, there would need to be recognition that many patients may not want to engage in ACP. In this way, ACP would be practiced as a distributed process of 'shared responsibility'. within which individuals would each see their own roles.

Within this framework, specialist palliative care could be considered as the specialists given that many already see them as the linchpin in ACP delivery. Yet without referral by another healthcare professional, and the integration of early palliative care alongside optimal disease-directed care, palliative care professionals will not see patients at the early stages of disease. Structures ensuring good communication links would need to be implemented to ensure that all healthcare professionals are aware of what patients have been told and understand about the implications of their disease. An awareness of these issues would provide healthcare professionals with a guide on how to approach the next stage of the discussion.

\section{CONCLUSION}

In busy clinical environments, ACP is uncommon for people with a brain tumour. This means that important decisions about end of life and future care might be overlooked and undocumented. 'Shared responsibility' has been interpreted as 'others' responsibility' laying the basis for a culture of avoidance of ACP. Through this, healthcare professionals can legitimate why they do not do ACP because they assume another member of the team will. One way to address this culture might be to explore a generalists and specialists model of ACP used in other models of integrated care. ${ }^{40-42}$ In this, particular healthcare professionals would be ascribed particular responsibilities. Healthcare professionals are in some ways already adopting this model. But without structure this is likely to fail given a tendency for people to assume the role of generalist. These roles would need to be delineated in codes of conduct and set within a formalised pathway to ensure patients are routinely offered ACP. Healthcare professionals whether generalist or specialist would need to be supported by focused training.

Acknowledgements The authors are very grateful to the healthcare professionals who gave their time to participate in this qualitative study. The authors would like to thank JN for his epidemiological advice and Sharon Scott for her assistance in the early stages of study design. The authors also acknowledge Marie Curie in their funding of the research department responsible for the execution of this study.

Contributors HL made substantial contributions to the design of the work, collection and interpretation of data, manuscript drafting and revision. JN made substantial contributions to the conception and design of the work, interpretation of data and manuscript revision. LT made substantial contributions to the conception and design of the work, interpretation of data and manuscript revision. EW made substantial contributions to the conception and design of the work, interpretation of data and manuscript revision. LJ made substantial contributions to the conception and design of the work, interpretation of data and manuscript revision. ELS made substantial contributions to the conception and design of the work, interpretation of data and manuscript revision. ET made substantial contributions to the conception and design of the work, interpretation of data and manuscript revision. JTSL made substantial contributions to the conception and design of the work, interpretation 
of data and manuscript revision. All authors gave final approval of the version to be published.

Funding This study is funded by The National Brain Appeal.

Competing interests None declared.

Patient consent Obtained.

Ethics approval Research and Development approval was obtained from UCLH Joint Research Office (\#12/0268).

Provenance and peer review Not commissioned; externally peer reviewed.

Data sharing statement Given the sensitive nature of the study interviews, raw data are not publicly available. Interested persons may contact the corresponding author for more information.

Open Access This is an Open Access article distributed in accordance with the Creative Commons Attribution Non Commercial (CC BY-NC 4.0) license, which permits others to distribute, remix, adapt, build upon this work non-commercially, and license their derivative works on different terms, provided the original work is properly cited and the use is non-commercial. See: http://creativecommons.org/ licenses/by-nc/4.0/

(c) Article author(s) (or their employer(s) unless otherwise stated in the text of the article) 2018. All rights reserved. No commercial use is permitted unless otherwise expressly granted.

\section{REFERENCES}

1. Cancer Research UK. Brain, other CNS and intracranial tumours (C70-C72, C75.1-C75.3, D32-D33, D35.2-D35.4, D42-D43, D44.3-D44.5): 2012-2014. http://www.cancerresearchuk.org/sites/ default/files/cstream-node/cases_crude_allbrain_i14.pdf (accessed May 2017).

2. Ferlay JSI, Ervik M, Dikshit R, et al. Cancer incidence and mortality worldwide: IARC cancer base No. 11. Lyon, France: International Agency for Research on Cancer, 2012. http://globocan.iarc.fr

3. Cancer Research UK. Malignant Brain, Other CNS and Intracranial Tumours (C70-C72, C75.1-C75.3): 2010-2012. http://www. cancerresearchuk.org/sites/default/files/cstream-node/inc anatomicalsite brain malignant.pdf (accessed May 2017).

4. Cancer Research UK. Brain cancer (C71): 2010-2011. http://www. cancerresearchuk.org/sites/default/files/cstream-node/surv_1_5_ 10yr_brain.pdf (accessed May 2017).

5. Cancer Research UK. Brain, other CNS and intracranial tumours mortality statistics. http://www.cancerresearchuk.org/healthprofessional/cancer-statistics/statistics-by-cancer-type/brain-othercns-and-intracranial-tumours/mortality (accessed May 2017).

6. Brain Tumour Research. Report on national research funding: Addressing the historic underfunding of brain tumour research. https://www.braintumourresearch.org/docs/default-source/defaultdocument-library/public-affairs-and-campaigning-documents/braintumour-research---report-on-national-research-funding---2016.pdf (accessed December 2017).

7. Molassiotis A, Wilson B, Brunton L, et al. Symptom experience in patients with primary brain tumours: a longitudinal exploratory study. Eur J Oncol Nurs 2010;14:410-6.

8. Walbert T, Khan M. End-of-life symptoms and care in patients with primary malignant brain tumors: a systematic literature review. $J$ Neurooncol 2014;117:217-24.

9. Philip J, Collins A, Brand CA, et al. "I'm just waiting...": an exploration of the experience of living and dying with primary malignant glioma. Support Care Cancer 2014;22:389-97.

10. Faithfull S, Cook K, Lucas C. Palliative care of patients with a primary malignant brain tumour: case review of service use and support provided. Palliat Med 2005;19:545-50.

11. Sizoo EM, Braam L, Postma TJ, et al. Symptoms and problems in the end-of-life phase of high-grade glioma patients. Neuro Oncol 2010;12:1162-6.

12. Halkett GK, Lobb EA, Oldham L, et al. The information and support needs of patients diagnosed with high grade glioma. Patient Educ Couns 2010;79:112-9.

13. Llewellyn H, Higgs P, Sampson EL, et al. Topographies of 'care pathways' and 'healthscapes': Reconsidering the multiple journeys of people with a brain tumour. Sociol Health IIIn 2017.

14. Moore G, Collins A, Brand C, et al. Palliative and supportive care needs of patients with high-grade glioma and their carers: a systematic review of qualitative literature. Patient Educ Couns 2013;91:141-53.
15. The General Medical Council. Treatment and care towards the end of life: good practice in decision making, 2010.

16. NHS End of Life Care. Advance care planning: a guide for health and social care staff, 2008.

17. Song K, Amatya B, Voutier C, et al. Advance care planning in patients with primary malignant brain tumors: a systematic review. Front Oncol 2016;6:223-37.

18. The Brain Tumour Trust Charity. Position paper: End of life care. https://www.thebraintumourcharity.org/media/filer_public/b5/46/ b5468651-6092-4e0c-b263-1d306232c90d/end_of_life_care_-_polic y_position_statement.pdf (accessed May 2017).

19. Henry C, Seymour J, Ryder S. Advance care planning: a quide for health and social care staff. London: Department of Health, 2007.

20. Mullick A, Martin J, Sallnow L. An introduction to advance care planning in practice. BMJ 2013;347:f6064-32.

21. Dening KH, Jones L, Sampson EL. Advance care planning for people with dementia: a review. Int Psychogeriatr 2011;23:1535-51

22. Jones L, Harrington J, Barlow CA, et al. Advance care planning in advanced cancer: can it be achieved? An exploratory randomized patient preference trial of a care planning discussion. Palliat Support Care 2011;9:3-13.

23. Luckett T, Sellars M, Tieman J, et al. Advance care planning for adults with CKD: a systematic integrative review. Am J Kidney Dis 2014;63:761-70.

24. Robinson L, Dickinson C, Bamford C, et al. A qualitative study: professionals' experiences of advance care planning in dementia and palliative care,'a good idea in theory but. Palliat Med 2013;27:401-8.

25. Musa I, Seymour J, Narayanasamy MJ, et al. A survey of older peoples' attitudes towards advance care planning. Age Ageing 2015;44:371-6.

26. Seymour J, Almack K, Kennedy S. Implementing advance care planning: a qualitative study of community nurses' views and experiences. BMC Palliat Care 2010;9:4.

27. Waldrop DP, Meeker MA. Communication and advanced care planning in palliative and end-of-life care. Nurs Outlook 2012;60:365-9.

28. Barnes K, Jones L, Tookman A, et al. Acceptability of an advance care planning interview schedule: a focus group study. Palliat Med 2007;21:23-8.

29. Yee A, Seow YY, Tan SH, et al. What do renal health-care professionals in Singapore think of advance care planning for patients with end-stage renal disease? Nephrology 2011;16:232-8.

30. De Vleminck A, Pardon K, Beernaert K, et al. Barriers to advance care planning in cancer, heart failure and dementia patients: a focus group study on general practitioners' views and experiences. PLOS One 2014;9:e84905

31. Lewis J, Ritchie J. Generalising from qualitative research. In: Qualitative research practice: a guide for social science students and researchers, 2003:263-86.

32. Castleberry A. NVivo 10 [software program]. Version 10. QSR International, AJPE: 2014.

33. Corbin J, Strauss A. Basics of qualitative research: techniques and procedures for developing grounded theory, 2008.

34. Cancer MJ. The annual review of anthropology. ;2016:45;251-66.

35. Kaufman S. And a time to die: how American hospitals shape the end of life: Simon and Schuster, 2005.

36. Kaufman SR. Ordinary medicine: extraordinary treatments, longer lives, and where to draw the line Health Affairs. Durham (NC): Duke University Press, 2015.

37. Round J, Llewellyn H. Living up to a good death: complexities and constraints in end of life choices. In: Round J, eds. Care at the end of life: an economic perspective. Cham: Springer International Publishing, 2016:193-205.

38. Walter T. Historical and cultural variants on the good death. $B M J$ 2003;327:218-20.

39. Wuensch A, Goelz T, Ihorst G, et al. Effect of individualized communication skills training on physicians' discussion of clinical trials in oncology: results from a randomized controlled trial. BMC Cancer 2017;17:264.

40. Stille CJ, Jerant A, Bell D, et al. Coordinating care across diseases, settings, and clinicians: a key role for the generalist in practice. Ann Intern Med 2005:142:700-8.

41. Martin GP, Currie G, Finn R. Reconfiguring or reproducing intraprofessional boundaries? Specialist expertise, generalist knowledge and the 'modernization' of the medical workforce. Soc Sci Med 2009;68:1191-8.

42. Bone $A E$, Morgan M, Maddocks $M$, et al. Developing a model of short-term integrated palliative and supportive care for frail older people in community settings: perspectives of older people, carers and other key stakeholders. Age Ageing 2016;45:863-73. 